\title{
Electroencephalographic Rhythms in Alzheimer's Disease
}

\author{
Roberta Lizio, ${ }^{1}$ Fabrizio Vecchio, ${ }^{2}$ Giovanni B. Frisoni, ${ }^{3}$ Raffaele Ferri, ${ }^{4}$ \\ Guido Rodriguez, ${ }^{5}$ and Claudio Babiloni ${ }^{6,7}$
}

${ }^{1}$ IRCCS San Raffaele Pisana, 00163 Rome, Italy

${ }^{2}$ A.Fa.R., Department of Neuroscience, Hospital Fatebenefratelli, Isola Tiberina, 00186 Rome, Italy

${ }^{3}$ IRCCS “S. Giovanni di Dio-F.B.F.", 25125 Brescia, Italy

${ }^{4}$ Department of Neurology, Oasi Institute for Research on Mental Retardation and Brain Aging (IRCCS), 94078 Troina, Italy

${ }^{5}$ Clinical Neurophysiology, Department of Endocrinological and Metabolic Sciences, University of Genoa, 16132 Genoa, Italy

${ }^{6}$ Department of Biomedical Sciences, University of Foggia, 71100 Foggia, Italy

${ }^{7}$ Casa di Cura San Raffaele Cassino, 03043 Cassino Frosinone, Italy

Correspondence should be addressed to Fabrizio Vecchio, fabrizio.vecchio@uniroma1.it

Received 14 December 2010; Accepted 13 March 2011

Academic Editor: Fabio Ferrarelli

Copyright () 2011 Roberta Lizio et al. This is an open access article distributed under the Creative Commons Attribution License, which permits unrestricted use, distribution, and reproduction in any medium, provided the original work is properly cited.

Physiological brain aging is characterized by synapses loss and neurodegeneration that slowly lead to an age-related decline of cognition. Neural/synaptic redundancy and plastic remodelling of brain networking, also due to mental and physical training, promotes maintenance of brain activity in healthy elderly subjects for everyday life and good social behaviour and intellectual capabilities. However, age is the major risk factor for most common neurodegenerative disorders that impact on cognition, like Alzheimer's disease (AD). Brain electromagnetic activity is a feature of neuronal network function in various brain regions. Modern neurophysiological techniques, such as electroencephalography (EEG) and event-related potentials (ERPs), are useful tools in the investigation of brain cognitive function in normal and pathological aging with an excellent time resolution. These techniques can index normal and abnormal brain aging analysis of corticocortical connectivity and neuronal synchronization of rhythmic oscillations at various frequencies. The present review suggests that discrimination between physiological and pathological brain aging clearly emerges at the group level, with suggested applications also at the level of single individual. The possibility of combining the use of EEG together with biological/neuropsychological markers and structural/functional imaging is promising for a low-cost, non-invasive, and widely available assessment of groups of individuals at-risk.

\section{Introduction}

Since its discovery and introduction, the electroencephalogram (EEG) was viewed with a great enthusiasm as the only methodology allowing a direct, online view of the "brain at work" [1]. The enormous complexity of the EEG signal should not surprise us since, the EEG is a direct correlate of brain function, and the brain is a complex system. So far, the EEG has been the most utilized signal to clinically monitor brain function. It offers appreciable promise as a means to characterize significant deviations from the "natural" aging found in Alzheimer and other dementias [2]. Since the 1970s, first with the introduction of structural imaging technologies such as computer-assisted tomography (CAT) and magnetic resonance imaging (MRI), and then with the development of regional metabolic-perfusion methods such as positron emission tomography (PET), single photon emission-computed tomography (SPECT), and the ability to map oxygen consumption and regional blood flow in specific neural locations with functional magnetic resonance imaging (fMRI), EEG has been supplanted in basic and clinical studies. These new techniques produce noninvasive views of in vivo brain anatomy with considerable resolution that contributed to their clinical and, therefore, economic utility. However, these functional brain imaging methods, despite their high spatial resolution for anatomical details, are relatively limited in their temporal resolution when measuring functional brain activation (seconds to minutes). Thus, these more recent neuroimaging techniques cannot discriminate the activation of different relays within a distributed network either in 
series or in parallel [3]. Over the years, several improvements have been introduced to EEG measures in part, because neuroelectric signals can track information processing with millisecond precision. Therefore, even if the EEG is affected by the problem of low spatial resolution when compared to other techniques (e.g., fMRI and PET), its high temporal resolution makes it possible to highlight the mechanism of temporal synchronization of the cortical pyramidal neurons. Compared to fMRI and PET, the advantage of using EEG is the possibility to evaluate the physiological mechanisms of cortical neural synchronization at the basis of the emerging brain feature: brain oscillations.

It should be noted that a high temporal resolution is crucial for the study of an emerging property of brain activity, namely, the spontaneous and event-related oscillatory activity at different frequencies ranging at $2-4 \mathrm{~Hz}$ (delta), 4-8 Hz (theta), $8-13 \mathrm{~Hz}$ (alpha), $13-30 \mathrm{~Hz}$ (beta), and $>30 \mathrm{~Hz}$ (gamma). Each of these frequencies conveys peculiar physiological information on brain functional state during sleep and wake periods.

Among the main purposes of modern neuroscientific research are the identification of patterns of neuronal activity underlying cognitive function and the finding of global functional indexes quick to be automatically computed towards clinical applications. It is, therefore, important to implement techniques that may measure natural brain aging and discriminate it from neurodegeneration $[4,5]$.

Recently, greater attention has been focused on the application of quantitative EEG (qEEG) and/or event-related potentials (ERPs) as suitable clinical markers of early stage of disease or its progression [6]. This is likely a result of recent improvements in the ease of the technology used and in the access to sufficient computing power and algorithms necessary for rapid processing of very complex raw datasets. Examples of recent technological advances include a reduction in the size (and portability) of EEG amplifiers and the development of high-density array nets that do not require skin abrasion to places with low impedance. It has been reported that a positive ERP peaking $600 \mathrm{~ms}$ after the zerotime of stimuli to be encoded (P600) was reduced in patients with Alzheimer's disease (AD) and mild cognitive impairment (MCI), particularly in those MCI patients who subsequently converted to $\mathrm{AD}[7,8]$. Furthermore, a positive ERP peaking $300 \mathrm{~ms}$ after the zerotime of oddball stimuli (P300) was found reduced in patients with dementia [6]. Thus, there exist theoretical and empirical reasons for the application of ERPs as a measure of individual variation of cognitive function along pathological aging [9]. It has been shown that it is sensitive to Alzheimer's disease processes during its early stages [9]. However, recording of ERPs requires a peculiar setup between the stimulation device and EEG machine, about 40-60 minutes of time for the examination in the patient, and technicians able to carry out engaging experimental conditions. In this regard, recording of resting state EEG rhythms represents a procedure much easier and rapid that does not require stimulation devices.

The present paper outlines the impact of EEG techniques for the measurement of physiological and pathological brain aging and provides a comprehensive analysis of brain aging by the analysis of resting state EEG rhythms in elderly subjects with various degrees of cognitive decline. Its major goal is to highlight the emerging neurophysiological findings important to determine whether these techniques provide sufficient innovative and potentially useful information for the assessment of normal aging and dementia, both at the group- and at the single-subject levels.

Furthermore, it is to underline the practical utility of the EEG technique as global functional indexes quickly evaluable for automatic computation towards clinical applications.

\section{Advanced EEG Techniques}

Advanced EEG analysis techniques can illustrate changes in specific rhythms oscillating at various frequencies over time, provide quantitative measurements of individual rhythms, and reduce the effects of volume currents from far-field generators $[10,11]$. Hence, EEG signals generated from extracerebral sources (e.g., electrocardiogram, electromyogram, electroretinogram, eye movement etc.) can be isolated from those produced by the brain, providing a direct measure of the recorded neuroelectric signals [11]. EEG coherence or synchronicity of rhythmic signals from separate electrodes, in different frequency bands, generated in different cortical areas, can also be measured.

The high-resolution EEG technique has markedly enhanced the spatial resolution of the conventional EEG from about $6-9 \mathrm{~cm}$ to $2-3 \mathrm{~cm}$ by the use of spatial enhancement methods such as Laplacian transformation with a regularized 3D spline function. This method reduces the low spatial EEG frequencies contributed by volume conduction and eliminates electrode reference influence [12-15]. Compared to other linear or nonlinear modelling analysis techniques of cortical sources of EEG-MEG, surface Laplacian estimation provides a rough representation of the neural currents without an explicit model of the generators (i.e., shape, number and location) by using a model of the head as a volume conductor [12, 13]. However, surface Laplacian methods cannot disentangle the activity of two spatially adjacent cortical zones such as primary somatosensory and motor areas that are contiguous across the central sulcus or deep cortical sources in secondary somatosensory and insular cortices. Surface Laplacian estimation is also unreliable when computed at the borders (i.e., temporo-parietal electrodes). Its maxima often overlie cortical sources of EEG potentials, since the influence of tangential relative to radial oriented generators is greater $[12,13,16]$.

Spectral coherence analysis indexes the temporal synchronization of two EEG time series among electrodes in the frequency domain and permits characterization of linear functional corticocortical connectivity. EEG spectral coherence is a normalized measure of the coupling between two electroencephalographic signals at any given frequency [17, 18]. It is commonly interpreted as an index of functional coupling $[19,20]$, mutual information exchange [17], functional coordination [21], and integrity of cortical neural pathways [22]. Its basic theoretical assumption is that when the activity of two cortical areas is functionally coordinated, 
the EEG rhythms of these cortical areas show linear correlation and high spectral coherence. In general, decreased coherence reflects reduced linear functional connections and information transfer (i.e., functional uncoupling) among cortical areas or modulation of common areas by a third region. In contrast, coherence increase is interpreted as augmented linear functional connections and information transfer (i.e., functional coupling), which reflects the interaction of different cortical structures for a given task. It has been repeatedly demonstrated that perceptive, cognitive, and motor processes are associated with enhanced EEG spectral coherence [23-26], as a function of the extension and type of the neural networks engaged $[27,28]$. Finally, the direction of the information flow within the EEG rhythms between pairs of electrodes can be estimated by a directed transfer function (DTF) [29-34].

There are different methods to solve the noninvasive localization of the neuronal generators responsible for measured EEG phenomena (i.e., the source reconstruction of the electromagnetic brain scalp signals). Low-resolution electromagnetic tomography algorithm (LORETA) software, which can be freely downloaded by Internet (http://www.unizh .ch/keyinst/NewLORETA/LORETA01.htm), has been successfully used in recent EEG studies on pathological brain aging [35-40]. LORETA computes 3D linear solutions (LORETA solutions) for the EEG inverse problem within a 3shell spherical head model including scalp, skull, and brain compartments [41-43].

LORETA solutions consisted of voxel z-current density values able to predict EEG spectral power density at scalp electrodes. As it is a reference-free method of EEG analysis, one can obtain the same LORETA source distribution for EEG data referenced to any reference electrode including common average. Furthermore, it can be also used from data collected by low spatial sampling (e.g., 19 electrodes) when cortical sources are estimated from resting EEG rhythms [44-47]. A normalization of the data was obtained by normalizing the LORETA current density at each voxel with the power density averaged across all frequencies (0.5$45 \mathrm{~Hz}$ ) and across all voxels of the brain volume. After the normalization, the solutions lost the original physical dimension and were represented by an arbitrary unit scale. This procedure reduced intersubjects variability and was used in previous EEG studies [36-40].

\section{Resting State EEG Rhythms and Physiological Aging}

Resting state cortical EEG rhythms typically change across physiological aging, with gradual modifications in profile and magnitude of the spectra power; in detail, it was observed a marked amplitude decrease of alpha $(8-13 \mathrm{~Hz})$ and a global "slowing" of the background EEG, which increases in power and spatial distribution in the slower delta $(2-4 \mathrm{~Hz})$ and theta $(4-8 \mathrm{~Hz})$ rhythms $[48-51]$. A recent study in a large sample of healthy subjects $(N=215,18-85$ years) confirmed an age-dependent power decrement of posterior low-frequency alpha (alpha $1 ; 8-10.5 \mathrm{~Hz}$ ) and delta rhythms [52].
Aging effects on parieto-occipital alpha rhythms presumably reflect the activity of dominant oscillatory neural network in the resting awaken brain. This activity is modulated by thalamocortical and corticocortical interactions facilitating/inhibiting the transmission of sensorimotor information and the retrieval of semantic information from cortical storage $[27,53,54]$.

In the condition of awaken rest, alpha 1 frequency would be mainly related to subject's global attentional readiness [54-58]. Noteworthy, there is consensus that alpha rhythms represent the dominant resting oscillations of the adult, awaken human brain [54-58] and have been linked to intelligent quotient, memory, and cognition [51]. Whereas high-frequency alpha rythms reflect the oscillation of specific neural systems for the elaboration of sensorimotor or semantic information $[50,55,56]$. Over the course of "natural" aging, the power decrease of the occipital alpha rhythms might be associated with changes in the cholinergic basal forebrain system function, which sustain the excitatory activity in the cholinergic brainstem pathway [59].

Neuroelectric output does not scale linearly with inputs received. Therefore, that assessment of nonlinear EEG interactions is important, as this method can provide information on the strength, direction, and topography of the interdependencies. Spatial organization of nonlinear interactions between different brain regions has been investigated to compare anterior-posterior intrahemispheric and left-right interhemispheric interactions across physiological aging. Differences were found in the rates of interdependencies between the left prefrontal and the right parietal regions between young and elderly, suggesting that the aging brain engages the right parietal region to assist the pre-frontal cortex [60].

\section{Resting State EEG Rhythms and Dementia}

Dementia is one of the most frequent chronic diseases of the elderly, and it is characterized by loss of intellectual and behavioral abilities that interfere with daily functioning. Dementia incidence tends to increase with age affecting over $30 \%$ of people after age $85[61,62]$. The elderly are the fastest growing segment of the population. Consequently, social costs for managing dementia are expected to rise becoming an important social problem. Furthermore, dementia profoundly affects the caregivers and family dynamics and relationships. Alzheimer's disease is the most common cause of dementia in geriatric patients.

Important neuropathological features indicating Alzheimer's dementia (AD) include brain cortical and subcortical atrophy leading to ventricular enlargements primarily due to neuronal loss in the temporal and parietal structures. Among the primary markers of Alzheimer's disease, microscopic signs including neurofibrillary tangles (intracellular aggregates of tau protein filaments) and amyloid plaques (extracellular aggregates of amyloid beta-peptides) that are dispersed throughout the cerebral cortex and basal ganglia, particularly concentrated in the hippocampus, entorhinal cortex, and postcentral parietal neocortex [63]. Tangles are 
mainly found in hippocampal and parahippocampal limbic structures, whereas amyloid plaques are largely diffuse throughout the cortex [64]. A neurophysiological hallmark of brain aging is a progressive impairment of use-dependent synaptic plasticity and of synaptic connectivity between neurons and its association with the degree of dementia [65]. However, in preclinical conditions, plastic compensatory remodelling appears to continue that maintains neural function so that the neuronal and synaptic death can occur in the absence of dementia symptoms for an unknown period of time that might take for years or decades.

When compared to the resting state EEG rhythms of healthy normal elderly (Nold) subjects, AD patients showed an amplitude increase of widespread delta and theta sources and an amplitude decrease of posterior alpha $(8-13 \mathrm{~Hz})$ and/or beta $(13-30 \mathrm{~Hz})$ sources [35, 47, 66-69]. The observation of these abnormalities of the EEG rhythms could allow a discrimination among different dementia diagnoses for instance, a marked decline of posterior slow-frequency alpha power shows peculiar features in mild AD subjects when compared to cerebrovascular dementia, frontotemporal dementia and normal elderly subjects with similar cognitive impairment. Furthermore, pathological increased amplitude of the theta sources characterized cerebrovascular dementia patients [47].

These EEG abnormalities have been associated with altered regional cerebral blood flow/metabolism and with impaired global cognitive function as evaluated by MMSE $[68,70-72]$.

Of note, early stages of $\mathrm{AD}$ (even preclinical) are typically associated with slowing down of resting occipital alpha rhythms, namely, a decrease of the individual alpha frequency (IAF) peak in power density [73]. The IAF peak, defined as the frequency associated with the strongest EEG power at the extended alpha range [51], should be always taken into account in EEG studies in AD subjects, since power changes in theta and alpha bands might be dependent phenomena. Furthermore, the conventional partition of EEG power into many conventional frequency bands allows the comparison of the results with those of most of the field studies but may prevent the separation of independent EEG rhythms or sources.

Despite the evidence of abnormal cortical rhythms in MCI and AD, EEG analysis alone is unable to allow a diagnosis of disease. Additional biological parameters are needed for this purpose. In this regard, several studies have shown a strict relationship between genetic risk factors such as Apolipoprotein E $\varepsilon 4$ genotype (Apo-E $\varepsilon 4$ ) and late-onset AD. Apo-E $\varepsilon 4$ has been found to affect EEG rhythms in AD patients, it is associated with abnormalities of resting state EEG rhythms in $\mathrm{AD}$ [74-76] with relatively specific EEG measures. Compared to $\mathrm{AD}$ patients with $\varepsilon 2$ and $\varepsilon 3, \mathrm{AD}$ patients with $\varepsilon 4$ showed higher theta and lower beta spectral power [75]. Furthermore, the AD ApoE $\varepsilon 4$ carriers patients were characterized by higher theta power and lower beta power at baseline, whereas they were characterized by higher delta power and lower alpha power at 3 years at followup [76]. Moreover, $\mathrm{AD}$ patients with $\mathrm{ApoE} \varepsilon 4$ has been related to selective decrease in functional corticocortical connectivity, which was suggested by the reduction of right and left temporoparietal, right temporofrontal, and left occipitoparietal alpha EEG coherence [74]. Thus, genetic risk factors for $\mathrm{AD}$ is combined with relatively specific EEG measures.

EEG power per se does not capture one of the main features of $\mathrm{AD}$, namely, the impairment of functional neural connectivity. It has been reported that $\mathrm{AD}$ patients present a reduced linear coupling of resting state EEG rhythms among cortical regions, as revealed by spectral EEG coherence $[22,74,77-80]$, suggesting a linear temporal synchronicity of coupled EEG rhythms from simultaneously engaged neural sources. Such findings imply that functional coupling of cortical rhythms at certain frequency bands might be interesting features of $\mathrm{AD}$ and that abnormality of cortical EEG coherence may be a fine-grained marker of $\mathrm{AD}$, which is supposed to reflect a disease of cerebral networks subserving global cognition. It could be speculated that this impaired pattern of EEG functional coupling is modulated by cholinergic systems and that a decrease of cortical EEG coherence is characterized by defective basal forebrain cholinergic inputs to cortex and hippocampus [81].

Most EEG studies of AD have reported a prominent decrease of alpha band coherence $[22,65,74,77-80,82-$ 85] This result also has been found to be associated with ApoE genetic risk, which is hypothesized to be mediated by cholinergic deficit [74]. However, delta and theta band coherence changes in $\mathrm{AD}$ are not homogeneous, as some studies demonstrate contradictory results with either a decrease or an increase of slow-band EEG coherence [22, 79, 82, 86]. These conflicting results might be due to the use of coherence markers from single electrode pairs rather than for the "total coherence" as obtained averaging the EEG spectral coherence across all combinations of electrode pairs. The latter may better take into account frequency band-by-frequency band the global impairment of brain networks and cognition along the $\mathrm{AD}$ process, which is supposed to be a disease affecting the functional integration within cerebral neural networks subserving cognition. In a recent study [87], the results show that the delta total coherence is higher in the AD than in the MCI and in the MCI than in the Nold group. Furthermore, the alphal total coherence is lower in the AD group than in the MCI and Nold groups. This evidence confirms that the functional coupling of resting EEG rhythms is progressively abnormal in amnesic MCI and AD subjects.

To improve the functional coupling evaluation, EEG and MEG data have been analyzed with procedures inspired by the theory of nonlinear dynamics, which provides a measure of signal dynamic coordination [88]. It is shown that $\mathrm{AD}$ patients generate a nonlinearly defined dimensional "complexity" of the EEG, which is a measure of signal dynamic coordination. The AD patients have significantly lower dimensional complexity of EEG than age-approximated non-demented controls. Thus it may be associated with deficient information processing in the brain injured by $\mathrm{AD}$. Brain rhythms loose the usual modulation in complexity as observed by eyes-open versus eyes-close comparisons, as a reflection of neuronal death, deficiency in neurotransmission, and/or loss of connectivity in local neuronal networks $[89,90]$. Nonlinear analysis has also been used to model 
brain flexibility in information processing, defined as the capability to affect state of information processing from identical initial conditions. AD patients show a decrease in information processing flexibility, such that EEG complexity decrease in $\mathrm{AD}$ might be attributable to decreased nonlinear dynamics that are associated with cognitive decline. Among the techniques for nonlinear brain dynamics, synchronization likelihood combines sensitivity to linear and nonlinear functional coupling of EEG/MEG rhythms [88]. This measure has been shown to be significantly decreased at alpha and low beta bands when comparing AD to MCI and/or Nold subjects [23, 91-93].

In addition to the corticocortical uncoupling progression, a decrease of synaptic coupling is likely to contribute to reducing selective EEG coherence for faster rhythms, as observed in healthy humans by transient use of a cholinergic synaptic blocker like scopolamine [94]. Animal models suggest that acetylcholine loss produces a decrease of highfrequency EEG couplings and an increase of slow-frequency couplings [95]. Loss or a significant drop in EEG synchronization in faster rhythms has also been correlated with decreased MMSE scores in MCI and AD patients [88]. Linear and Nonlinear EEG analyses improve classification accuracy of $\mathrm{AD}$ compared to unaffected controls, and these methods correlate with disease severity $[23,88,91]$.

Few studies have assessed EEG measures over the course of dementia progression. A significant increase of delta and theta power in conjunction with decrease of alpha and beta power over a period of 30 months from diagnosis have been found [96]. The length of the followup is of paramount importance and indicates the reason for a lack of findings over a 12-month period [97]. The major question in this context is "Which is the physiological mechanism at the basis of abnormal resting brain rhythms in MCI and AD?" Abnormality of resting EEG rhythms may originate from impairment in the cholinergic neural projections from basal forebrain, which is a pivotal aspect of AD [98]. Resting EEG alpha power is decreased from experimental damage to this cholinergic pathway [99]. Furthermore, the cholinergic basal forebrain has been found to be responsive to the treatment with cholinesterase inhibitors more for $\mathrm{AD}$ than other dementias [100]. Conversely, brainstem cholinergic innervations of the thalamus are relatively spared in $\mathrm{AD}$ patients [98]. Long-term (1 year) treatments of acetylcholinesterase inhibitors (AChEI) demonstrate less temporal and occipital alpha reduction for responders compared to nonresponders and a combined effect on delta and low alpha $[37,101]$. Hence, increasing cholinergic tone was related to restoring temporal and occipital alpha rhythms in responders. Brain cholinergic systems also appear to improve primarily cerebral blood flow with a functional impact on attentional and memory functions [102].

\section{Resting State EEG Rhythms and Mild Cognitive Impairment}

Assessing preclinical dementia is of keen interest as a clinical research issue, since MCI often precedes frank dementing illness. As the selective cognitive impairments characteristic of MCI are primarily memory-related and not severe enough to exceed standard clinical criteria for $\mathrm{AD}$, their prodromal qualities do not greatly impair daily functioning and can be identified by refined clinical and neuropsychological evaluation. Consistent MCI symptoms 3-5 years following their identification either remain stable or decrease in $30 \%-$ $50 \%$ of the cases, whereas the remaining cases progress toward a frank $\mathrm{AD}$ condition or, less frequently, to other dementias. The MCI condition has often been considered a precursor of AD despite the fact that not all the MCI patients develop the Alzheimer disease. Epidemiological and clinical followup studies confirm that MCI reflects a transition state towards mild $\mathrm{AD}$ and prompts the idea that early identification of MCI patients can facilitate rehabilitative or pharmacological interventions to slow down the disease progression [103-105]. Figure 1 illustrates MCI effects for low-frequency alpha $(8-10.5 \mathrm{~Hz})$ activity from parietal, occipital, and limbic areas that demonstrate an intermediate magnitude in MCI compared to mild $\mathrm{AD}$ and normal elderly [38]. Increase of slow EEG power coupled with a decrease in alpha activity is linked to cognitive performance decline in MCI compared to Nold. More important, the spectral magnitude of these sources is correlated negatively with MMSE scores across subjects of the three groups, suggesting that EEG evidence of alpha power decrease in MCI compared to normal subjects is related to behavioral cognition [66, $84,106-109]$. The relative spectral magnitude decrease of posterior low-frequency alpha sources in MCI may be related to an initial selective impairment of the cholinergic basal forebrain, which could induce a sustained increase of the excitatory activity in the cholinergic brainstem pathway [59, $94,95]$. TMS studies indicate that the cortex of AD patients is hyperexcitable and that such hyperexcitability even may offer clues for the differential diagnosis from other dementias in which the cholinergic deficit is not predominant.

As a consequence, the increased excitability of thalamocortical connections would desynchronize the resting alpha rhythms and enhance the cortical excitability.

Hence, changes of low-frequency alpha power in MCI and mild $\mathrm{AD}$ suggest a progressive impairment of the thalamocortical and corticocortical systems that govern visual attention. This hypothesis is consistent with clinical findings of increasing deficits of visuospatial abilities in MCI and mild AD [110]. Similarly, limbic sources imply a progressive impairment of thalamocortical and corticocortical systems regulating attention tone for memory functions.

Decreases in corticothalamic modulation and increase of slow EEG rhythms correlated to progressive cortical hypoperfusion have been found in $\mathrm{AD}[72,111]$. Abnormal delta and alpha sources in the posterior brain regions could, therefore, index the progressive decline of cognitive visuospatial functions across $\mathrm{MCI}$ and mild $\mathrm{AD}$ thereby supporting a transition between these conditions [103105]. An intriguing aspect includes the peculiar magnitude increase of the parieto-occipital high-frequency alpha sources (alpha 2, 10.5-13 Hz) in MCI compared to mild $\mathrm{AD}$ and normal elderly [38]. Furthermore, prospective studies have demonstrated that increased delta/theta activity, 


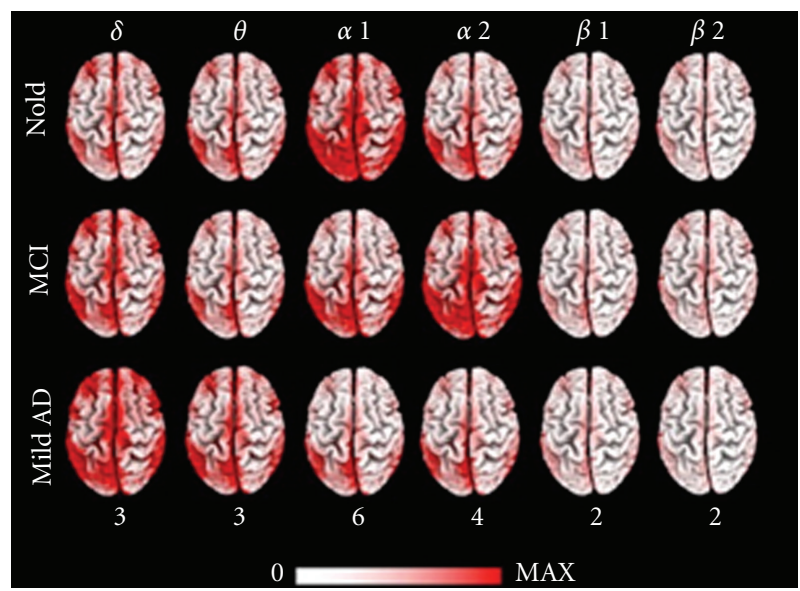

FIGURE 1: Grand average of low-resolution brain electromagnetic source tomography (LORETA) solutions (i.e., normalized relative current density at the cortical voxels) modeling the distributed EEG sources for delta, theta, alpha 1, alpha 2, beta 1 (13$20 \mathrm{~Hz})$, and beta $2(20-30 \mathrm{~Hz})$ bands in normal elderly (Nold), mild cognitive impairment (MCI) and mild Alzheimer's disease (AD) groups. The left side of the maps (top view) corresponds to the left hemisphere. Legend: LORETA, low-resolution brain electromagnetic tomography. Color scale: all power estimates were scaled based on the averaged maximum value (i.e., alpha 1 power value of occipital region in Nold). The maximal value of power is reported under each column.

decreased alpha and beta, and slowed mean frequency may be predictors of progression from MCI to dementia [66, 84]. These findings imply that neuroelectric indices could be developed for the preclinical assessment of dementia, as their acquisition are inexpensive, easily implemented, entirely non-invasive, and very well suited for large-scale screening and followup of at-risk populations. The hypothesis that presence of ApoE $\varepsilon 4$ affects sources of resting EEG rhythms in $\mathrm{MCI}$ and $\mathrm{AD}$ was assessed in $89 \mathrm{MCI}$ with $34.8 \% \varepsilon 4$ incidence and $103 \mathrm{AD}$ with $50.4 \% \varepsilon 4$ incidence [112]. Alpha 1 and 2 sources in occipital, temporal, and limbic areas were of lower amplitude in subjects carrying the ApoE $\varepsilon 4$ allele. For AD homozygous for ApoE $\varepsilon 4$ allele, abnormal temporo-parietal and occipitoparietal EEG or MEG rhythms were found [74, 88]. However, in addition to ApoE $\varepsilon 4$ allele, another important genetic risk factor for late-onset $\mathrm{AD}$ is haplotype $\mathrm{B}$ of CST3 (the gene coding for cystatin C-a neurotrophic protein), which was investigated to establish eventual links with cortical rhythmicity [113]. EEG measures were obtained from $84 \mathrm{MCI}$ with $42 \%$ B haplotype and $65 \mathrm{AD}$ with $40 \% \mathrm{~B}$ haplotype. Slow alpha (from parietal, occipital, and temporal areas) and fast alpha (from occipital areas) power were statistically lower in CST3 B carriers. A trend was observed for occipital delta power sources as stronger in CST3 B carriers than in noncarriers for both $\mathrm{MCI}$ and $\mathrm{AD}$ patients.

Association between the presence and amount of hippocampus atrophy in $\mathrm{AD}$ and $\mathrm{MCI}$ subjects and changes in sources of posterior slow rhythms have been observed by EEG and whole-head MEG [114-116]. Less known is the relationships between impairment of white matter and slow rhythms across the continuum from MCI to AD. This issue has been addressed with EEG assessments in MCI $(N=$ $34)$ and $\mathrm{AD}(N=65)$ cases [36]. Delta activity was related to the amount of cortical atrophy revealed by MRI voxelto-voxel volumetry of lobar brain volume (white and gray matter) such that as delta power increased, brain volume decreased. Thus, changes in brain structure and function could be found for MCI and AD patients.

As life expectancy and elderly populations in Western countries are increasing, the incidence of MCI that may predict $\mathrm{AD}$ or vascular dementia is rising. Cognitive impairment associated with MCI or $\mathrm{AD}$ is associated with decreased power and coherence in the alpha/beta band, at least at the group level. This observation suggests the occurrence of a functional disconnection among cortical areas, since both power and coherence in the delta and theta bands increase with cortical deafferentiation from subcortical structures [117]. However, the extent to which features of neuroelectric activity can be used to predict the conversion from MCI to $\mathrm{AD}$ in single subjects is as yet unclear. In a seminal EEG study, a multiple logistic regression of theta power (3.5$7.5 \mathrm{~Hz}$ ), mean frequency, and interhemispheric coherence has been able to to predict decline from MCI to $\mathrm{AD}$ at long term for with an overall predictive accuracy of about $90 \%$ [118]. Furthermore, spectral EEG coherence or other EEG features have shown to contribute to the discrimination of Nold from mild AD with $89 \%-45 \%$ of success, from MCI to $\mathrm{AD}$ with $92 \%-78 \%$ of success, and the conversion of MCI subjects to AD with $87 \%-60 \%$ of success $[66,79,84,119$ 124]. These findings are encouraging for future development of this prognostic and perhaps diagnostic approach [125].

Rossini et al. [106] investigated whether combined analysis of EEG power and coherence provide early and reliable discrimination of MCI subjects who will convert to $\mathrm{AD}$ after a relatively brief followup. Cortical connectivity using spectral coherence measures and LORETA was evaluated to characterize EEG sources at baseline in $69 \mathrm{MCI}$ cases that were reassessed clinically after about 14 months. At followup, 45 subjects were classified as stable MCI (MCI Stable), whereas the remaining 24 had converted to $\mathrm{AD}$ (MCI Converted). Results showed that at baseline, frontoparietal midline coherence as well as delta (temporal), theta (parietal, occipital, and temporal), and low-frequency alpha (central, parietal, occipital, temporal, and limbic) sources were stronger in MCI converted than MCI stable subjects. Cox regression modeling showed low midline coherence, and weak temporal source was associated with $10 \%$ annual rate $\mathrm{AD}$ conversion, while this rate increased up to $40 \%$ and $60 \%$ when strong temporal delta source and high midline gamma coherence were observed, respectively. This outcome indicates that quantitative EEG is able to predict with a good approximation $\mathrm{MCI}$ progression to $\mathrm{AD}$ in the short run.

\section{Conclusions}

The present paper highlights the use of modern EEG techniques that report assessment of physiological and pathological brain aging. Application of these techniques allows 
the quantification of the power and functional coupling of resting state EEG rhythms at scalp electrodes and mathematical cortical sources. The results reviewed in the present paper suggest that these quantitative indexes of resting state EEG rhythms might reflect neurodegenerative processes along preclinical and clinical stages of AD. Moreover, risk factors including genetic causes correlate with neurophysiological findings to reinforce their causative role in diagnosis and prognosis of pathologic brain aging. Unfortunately, this remarkable literature suffers from the partial lack of integration of various EEG techniques such as analysis of power density and functional coupling (i.e., spectral coherence, and directed transfer function) within a unique frame of goaldirected test for evaluation of physiological brain aging and discrimination from abnormal scenarios heralding neurodegeneration. In the near future, systematic evaluation of $\mathrm{AD}$ and other dementing disorders relative to normal aging using refined and integrated EEG techniques will help to coalesce these methodologies and improve diagnostic utility. If this approach can provide clinically useful information at the individual level, such methods should prompt design of an instrument widely available for large-scale population-based screening studies. Future studies should find which are qEEG markers for early diagnosis, prognosis, and monitoring of Alzheimer disease and explore the clinical utility of this methodological approach. The global structural and functional indexes are quick to be automatically computed towards clinical applications.

\section{References}

[1] H. U. Berger, "Über das elektrenkephalogramm des menschen," Archiv für Psychiatrie und Nervenkrankheiten, vol. 87, no. 1, pp. 527-570, 1929.

[2] H. Berger, Das Elektroenkephalogramm des Menschen. Halle an der Saale, , vol. 6 of Nova Acta Leopoldina, Buchdruckerei des Waisenhauses, Malbork, Poland, 1938.

[3] P. M. Rossini and G. Dal Forno, "Integrated technology for evaluation of brain function and neural plasticity," Physical Medicine and Rehabilitation Clinics of North America, vol. 15, no. 1, pp. 263-306, 2004.

[4] G. G. Celesia, D. Kaufman, and S. Cone, "Effects of age and sex on pattern electroretinograms and visual evoked potentials," Electroencephalography and Clinical Neurophysiology, vol. 68, no. 3, pp. 161-171, 1987.

[5] P. M. Rossini, "Chapter 6 implications of brain plasticity to brain-machine interfaces operation. A potential paradox?" International Review of Neurobiology, vol. 86, pp. 81-90, 2009.

[6] P. M. Rossini, S. Rossi, C. Babiloni, and J. Polich, "Clinical neurophysiology of aging brain: from normal aging to neurodegeneration," Progress in Neurobiology, vol. 83, no. 6, pp. 375-400, 2007.

[7] J. M. Olichney, S. K. Morris, C. Ochoa et al., "Abnormal verbal event related potentials in mild cognitive impairment and incipient Alzheimer's disease," Journal of Neurology Neurosurgery and Psychiatry, vol. 73, no. 4, pp. 377-384, 2002.

[8] J. M. Olichney, V. J. Iragui, D. P. Salmon, B. R. Riggins, S. K. Morris, and M. Kutas, "Absent event-related potential (ERP) word repetition effects in mild Alzheimer's disease," Clinical Neurophysiology, vol. 117, no. 6, pp. 1319-1330, 2006.

[9] J. Polich and J. Corey-Bloom, "Alzheimer's disease and P300: review and evaluation of task and modality," Current Alzheimer Research, vol. 2, no. 5, pp. 515-525, 2005.

[10] P. L. Nunez, "Toward a quantitive description of large-scale neocortical dynamic function and EEG," Behavioral and Brain Sciences, vol. 23, no. 3, pp. 371-398+432, 2000.

[11] F. Babiloni, F. Carducci, F. Cincotti et al., "Linear inverse source estimate of combined EEG and MEG data related to voluntary movements," Human Brain Mapping, vol. 14, no. 4, pp. 197-209, 2001.

[12] F. Babiloni, C. Babiloni, L. Fattorini, F. Carducci, P. Onorati, and A. Urbano, "Performances of surface Laplacian estimators: a study of simulated and real scalp potential distributions," Brain Topography, vol. 8, no. 1, pp. 35-45, 1995.

[13] F. Babiloni, C. Babiloni, F. Carducci, L. Fattorini, P. Onorati, and A. Urbano, "Spline Laplacian estimate of EEG potentials over a realistic magnetic resonance-constructed scalp surface model," Electroencephalography and Clinical Neurophysiology, vol. 98, no. 4, pp. 363-373, 1996.

[14] F. Babiloni, C. Babiloni, F. Carducci, F. Cincotti, and P. M. Rossini, "'The stone of madness' and the search for the cortical sources of brain diseases with non-invasive EEG techniques," Clinical Neurophysiology, vol. 114, no. 10, pp. 1775-1780, 2003.

[15] P. L. Nunez, Srinivasan R. Electric Fields of the Brain: The Neurophysics of EEG, Oxford University Press, New York, NY, USA, 2006.

[16] P. L. Nunez, Neocortical Dynamics and Human EEG Rhythms, Oxford University Press, New York, NY, USA, 1995.

[17] R. Rappelsberger and H. Petsche, "Probability mapping: power and coherence analyses of cognitive processes," Brain Topography, vol. 1, no. 1, pp. 46-54, 1988.

[18] C. Andrew and G. Pfurtscheller, "Event-related coherence as a tool for studying dynamic interaction of brain regions," Electroencephalography and Clinical Neurophysiology, vol. 98, no. 2, pp. 144-148, 1996.

[19] C. Gerloff, J. Richard, J. Hadley, A. E. Schulman, M. Honda, and M. Hallett, "Functional coupling and regional activation of human cortical motor areas during simple, internally paced and externally paced finger movements," Brain, vol. 121, no. 8, pp. 1513-1531, 1998.

[20] R. W. Thatcher, P. J. Krause, and M. Hrybyk, "Corticocortical associations and EEG coherence: a two-compartmental model," Electroencephalography and Clinical Neurophysiology, vol. 64, no. 2, pp. 123-143, 1986.

[21] A. Gevins, M. E. Smith, H. Leong et al., "Monitoring working memory load during computer-based tasks with EEG pattern recognition methods," Human Factors, vol. 40, no. 1, pp. 7991, 1998.

[22] T. Locatelli, M. Cursi, D. Liberati, M. Franceschi, and G. Comi, "EEG coherence in Alzheimer's disease," Electroencephalography and Clinical Neurophysiology, vol. 106, no. 3, pp. 229-237, 1998.

[23] C. Babiloni, R. Ferri, G. Binetti et al., "Fronto-parietal coupling of brain rhythms in mild cognitive impairment: a multicentric EEG study," Brain Research Bulletin, vol. 69, no. 1, pp. 63-73, 2006.

[24] P. Sauseng, W. Klimesch, M. Schabus, and M. Doppelmayr, "Fronto-parietal EEG coherence in theta and upper alpha 
reflect central executive functions of working memory," International Journal of Psychophysiology, vol. 57, no. 2, pp. 97-103, 2005.

[25] F. Vecchio, C. Babiloni, F. Ferreri et al., "Mobile phone emission modulates inter-hemispheric functional coupling of EEG alpha rhythms in elderly compared to young subjects," Clinical Neurophysiology, vol. 121, no. 2, pp. 163$171,2010$.

[26] F. Vecchio, C. Babiloni, F. Ferreri et al., "Mobile phone emission modulates interhemispheric functional coupling of EEG alpha rhythms," European Journal of Neuroscience, vol. 25, no. 6, pp. 1908-1913, 2007.

[27] G. Pfurtscheller and F. H. Lopes da Silva, "Event-related EEG/MEG synchronization and desynchronization: basic principles," Clinical Neurophysiology, vol. 110, no. 11, pp. 1842-1857, 1999.

[28] A. von Stein and J. Sarnthein, "Different frequencies for different scales of cortical integration: from local gamma to long range alpha/theta synchronization," International Journal of Psychophysiology, vol. 38, no. 3, pp. 301-313, 2000.

[29] M. J. Kaminski and K. J. Blinowska, "A new method of the description of the information flow in the brain structures," Biological Cybernetics, vol. 65, no. 3, pp. 203-210, 1991.

[30] M. J. Kamiński, K. J. Blinowska, and W. Szelenberger, "Topographic analysis of coherence and propagation of EEG activity during sleep and wakefulness," Electroencephalography and Clinical Neurophysiology, vol. 102, no. 3, pp. 216227, 1997.

[31] A. Korzeniewska, S. Kasicki, M. Kamiński, and K. J. Blinowska, "Information flow between hippocampus and related structures during various types of rat's behavior," Journal of Neuroscience Methods, vol. 73, no. 1, pp. 49-60, 1997.

[32] T. Mima, T. Matsuoka, and M. Hallett, "Functional coupling of human right and left cortical motor areas demonstrated with partial coherence analysis," Neuroscience Letters, vol. 287, no. 2, pp. 93-96, 2000.

[33] C. Babiloni, F. Babiloni, F. Carducci et al., "Coupling between "Hand" primary sensorimotor cortex and lower limb muscles after ulnar nerve surgical transfer in paraplegia," Behavioral Neuroscience, vol. 118, no. 1, pp. 214-222, 2004.

[34] C. Babiloni, F. Carducci, F. Vecchio et al., "Functional frontoparietal connectivity during short-term memory as revealed by high-resolution EEG coherence analysis," Behavioral Neuroscience, vol. 118, no. 4, pp. 687-697, 2004.

[35] T. Dierks, V. Jelic, R. D. Pascual-Marqui et al., "Spatial pattern of cerebral glucose metabolism (PET) correlates with localization of intracerebral EEG-generators in Alzheimer's disease," Clinical Neurophysiology, vol. 111, no. 10, pp. 18171824, 2000.

[36] C. Babiloni, G. Frisoni, M. Steriade et al., "Frontal white matter volume and delta EEG sources negatively correlate in awake subjects with mild cognitive impairment and Alzheimer's disease," Clinical Neurophysiology, vol. 117, no. 5, pp. 1113-1129, 2006.

[37] C. Babiloni, E. Cassetta, G. Dal Forno et al., "Donepezil effects on sources of cortical rhythms in mild Alzheimer's disease: responders vs. Non-responders," NeuroImage, vol. 31, no. 4, pp. 1650-1665, 2006.

[38] C. Babiloni, G. Binetti, E. Cassetta et al., "Sources of cortical rhythms change as a function of cognitive impairment in pathological aging: a multicenter study," Clinical Neurophysiology, vol. 117, no. 2, pp. 252-268, 2006.
[39] C. Babiloni, R. Lizio, F. Vecchio et al., "Reactivity of cortical alpha rhythms to eye opening in mild cognitive impairment and Alzheimer's disease: an EEG study," Journal of Alzheimer's Disease, vol. 22, no. 4, pp. 1047-1064, 2010.

[40] C. Babiloni, G. B. Frisoni, F. Vecchio et al., "Stability of clinical condition in mild cognitive impairment is related to cortical sources of alpha rhythms: an electroencephalographic study," Human Brain Mapping, 2010. In press.

[41] R. D. Pascual-Marqui, C. M. Michel, and D. Lehmann, "Low resolution electromagnetic tomography: a new method for localizing electrical activity in the brain," International Journal of Psychophysiology, vol. 18, no. 1, pp. 49-65, 1994.

[42] R. D. Pascual-Marqui, D. Lehmann, T. Koenig et al., "Low resolution brain electromagnetic tomography (LORETA) functional imaging in acute, neuroleptic-naive, first-episode, productive schizophrenia," Psychiatry Research, vol. 90, no. 3, pp. 169-179, 1999.

[43] R. D. Pascual-Marqui, M. Esslen, K. Kochi, and D. Lehmann, "Functional imaging with low-resolution brain electromagnetic tomography (LORETA): a review," Methods and Findings in Experimental and Clinical Pharmacology, vol. 24, pp. 91-95, 2002.

[44] M. Saletu, P. Anderer, G. M. Saletu-Zyhlarz et al., "EEGtomographic studies with LORETA on vigilance differences between narcolepsy patients and controls and subsequent double-blind, placebo-controlled studies with modafinil," Journal of Neurology, vol. 251, no. 11, pp. 1354-1363, 2004.

[45] T. Isotani, H. Tanaka, D. Lehmann et al., "Source localization of EEG activity during hypnotically induced anxiety and relaxation," International Journal of Psychophysiology, vol. 41, no. 2, pp. 143-153, 2001.

[46] S. Mientus, J. Gallinat, Y. Wuebben et al., "Cortical hypoactivation during resting EEG in schizophrenics but not in depressives and schizotypal subjects as revealed by low resolution electromagnetic tomography (LORETA)," Psychiatry Research, vol. 116, no. 1-2, pp. 95-111, 2002.

[47] C. Babiloni, G. Binetti, E. Cassetta et al., "Mapping distributed sources of cortical rhythms in mild Alzheimer's disease. A multicentric EEG study," NeuroImage, vol. 22, no. 1, pp. 57-67, 2004.

[48] K. Dujardin, J. L. Bourriez, and J. D. Guieu, "Event-related desynchronization (ERD) patterns during verbal memory tasks: effect of age," International Journal of Psychophysiology, vol. 16, no. 1, pp. 17-27, 1994.

[49] K. Dujardin, J. L. Bourriez, and J. D. Guieu, "Eventrelated desynchronization (ERD) patterns during memory processes: effects of aging and task difficulty," Electroencephalography and Clinical Neurophysiology, vol. 96, no. 2, pp. 169-182, 1995.

[50] D. W. Klass and R. P. Brenner, "Electroencephalography of the elderly," Journal of Clinical Neurophysiology, vol. 12, no. 2, pp. 116-131, 1995.

[51] W. Klimesch, "EEG alpha and theta oscillations reflect cognitive and memory performance: a review and analysis," Brain Research Reviews, vol. 29, no. 2-3, pp. 169-195, 1999.

[52] C. Babiloni, G. Binetti, A. Cassarono et al., "Sources of cortical rhythms in adults during physiological aging: a multicentric EEG study," Human Brain Mapping, vol. 27, no. 2, pp. 162-172, 2006.

[53] C. H. M. Brunia, "Neural aspects of anticipatory behavior," Acta Psychologica, vol. 101, no. 2-3, pp. 213-242, 1999.

[54] M. Steriade and R. R. Llinas, "The functional states of the thalamus and the associated neuronal interplay," Physiological Reviews, vol. 68, no. 3, pp. 649-742, 1988. 
[55] W. Klimesch, "Memory processes, brain oscillations and EEG synchronization," International Journal of Psychophysiology, vol. 24, no. 1-2, pp. 61-100, 1996.

[56] W. Klimesch, "EEG-alpha rhythms and memory processes," International Journal of Psychophysiology, vol. 26, no. 1-3, pp. 319-340, 1997.

[57] W. Klimesch, M. Doppelmayr, H. Russegger, T. Pachinger, and J. Schwaiger, "Induced alpha band power changes in the human EEG and attention," Neuroscience Letters, vol. 244, no. 2, pp. 73-76, 1998.

[58] P. M. Rossini, M. T. Desiato, F. Lavaroni, and M. D. Caramia, "Brain excitability and electroencephalographic activation: non-invasive evaluation in healthy humans via transcranial magnetic stimulation," Brain Research, vol. 567, no. 1, pp. 111-119, 1991.

[59] M. Sarter and J. P. Bruno, "Cortical acetylcholine, reality distortion, schizophrenia, and Lewy Body Dementia: too much or too little cortical acetylcholine?" Brain and Cognition, vol. 38, no. 3, pp. 297-316, 1998.

[60] J. R. Terry, C. Anderson, and J. A. Horne, "Nonlinear analysis of EEG during NREM sleep reveals changes in functional connectivity due to natural aging," Human Brain Mapping, vol. 23, no. 2, pp. 73-84, 2004.

[61] B. A. Vicioso, "Dementia: when is it not Alzheimer disease?" American Journal of the Medical Sciences, vol. 324, no. 2, pp. 84-95, 2002.

[62] A. B. Graves and W. A. Kukull, "The epidemiology of dementia," in Handbook of Dementing Illnesses, J. C. Morris, Ed., pp. 23-69, Marcel Dekker, New York, NY, USA, 1994.

[63] K. Iqbal, A. D. C. Alonso, E. El-Akkad et al., "Significance and mechanism of Alzheimer neurofibrillary degeneration and therapeutic targets to inhibit this lesion," Journal of Molecular Neuroscience, vol. 19, no. 1-2, pp. 95-99, 2002.

[64] M. Goedert and M. G. Spillantini, "A century of Alzheimer's disease," Science, vol. 314, no. 5800, pp. 777-781, 2006.

[65] I. A. Cook and A. F. Leuchter, "Synaptic dysfunction in Alzheimer's disease: clinical assessment using quantitative EEG," Behavioural Brain Research, vol. 78, no. 1, pp. 15-23, 1996.

[66] C. Huang, L. O. Wahlund, T. Dierks, P. Julin, B. Winblad, and V. Jelic, "Discrimination of Alzheimer's disease and mild cognitive impairment by equivalent EEG sources: a crosssectional and longitudinal study," Clinical Neurophysiology, vol. 111, no. 11, pp. 1961-1967, 2000.

[67] N. V. Ponomareva, N. D. Selesneva, and G. A. Jarikov, "EEG alterations in subjects at high familial risk for Alzheimer's disease," Neuropsychobiology, vol. 48, no. 3, pp. 152-159, 2003.

[68] J. Jeong, "EEG dynamics in patients with Alzheimer's disease," Clinical Neurophysiology, vol. 115, no. 7, pp. 14901505, 2004.

[69] L. S. Prichep, "Use of normative databases and statistical methods in demonstrating clinical utility of QEEG: importance and cautions," Clinical EEG and Neuroscience, vol. 36, no. 2, pp. 82-87, 2005.

[70] E. P. Sloan, G. W. Fenton, N. S. J. Kennedy, and J. M. MacLennan, "Electroencephalography and single photon emission computed tomography in dementia: a comparative study," Psychological Medicine, vol. 25, no. 3, pp. 631-638, 1995.

[71] G. Rodriguez, P. Vitali, and F. Nobili, "Long-term effects of boxing and judo-choking techniques on brain function," Italian Journal of Neurological Sciences, vol. 19, no. 6, pp. 367372, 1998.
[72] G. Rodriguez, F. Nobili, F. Copello et al., "Tc-HMPAO regional cerebral blood flow and quantitative electroencephalography in Alzheimer's disease: a correlative study," Journal of Nuclear Medicine, vol. 40, no. 4, pp. 522-529, 1999.

[73] D. V. Moretti, C. Babiloni, G. Binetti et al., "Individual analysis of EEG frequency and band power in mild Alzheimer's disease," Clinical Neurophysiology, vol. 115, no. 2, pp. 299308, 2004.

[74] V. Jelic, P. Julin, M. Shigeta et al., "Apolipoprotein E $\varepsilon 4$ allele decreases functional connectivity in Alzheimer's disease as measured by EEG coherence," Journal of Neurology Neurosurgery and Psychiatry, vol. 63, no. 1, pp. 59-65, 1997.

[75] M. Lehtovirta, J. Partanen, M. Könönen et al., "Spectral analysis of EEG in Alzheimer's disease: relation to apolipoprotein E polymorphism," Neurobiology of Aging, vol. 17, no. 4, pp. 523-526, 1996.

[76] M. Lehtovirta, J. Partanen, M. Könönen et al., "A longitudinal quantitative EEG study of Alzheimer's disease: relation to apolipoprotein E polymorphism," Dementia and Geriatric Cognitive Disorders, vol. 11, no. 1, pp. 29-35, 2000.

[77] Y. Wada, Y. Nanbu, Y. Koshino, N. Yamaguchi, and T. Hashimoto, "Reduced interhemispheric EEG coherence in Alzheimer disease: analysis during rest and photic stimulation," Alzheimer Disease and Associated Disorders, vol. 12, no. 3, pp. 175-181, 1998.

[78] V. Knott, E. Mohr, C. Mahoney, and V. Ilivitsky, "Electroencephalographic coherence in Alzheimer's disease: comparisons with a control group and population norms," Journal of Geriatric Psychiatry and Neurology, vol. 13, no. 1, pp. 1-8, 2000.

[79] G. Adler, S. Brassen, and A. Jajcevic, "EEG coherence in Alzheimer's dementia," Journal of Neural Transmission, vol. 110, no. 9, pp. 1051-1058, 2003.

[80] Y. Wada, Y. Nanbu, M. Kikuchi, Y. Koshino, T. Hashimoto, and N. Yamaguchi, "Abnormal functional connectivity in Alzheimer's disease: intrahemispheric EEG coherence during rest and photic stimulation," European Archives of Psychiatry and Clinical Neuroscience, vol. 248, no. 4, pp. 203-208, 1998.

[81] C. J. Stam, B. F. Jones, I. Manshanden et al., "Magnetoencephalographic evaluation of resting-state functional connectivity in Alzheimer's disease," NeuroImage, vol. 32, no. 3, pp. 1335-1344, 2006.

[82] A. F. Leuchter, J. E. Spar, D. O. Walter, and H. Weiner, "Electroencephalographic spectra and coherence in the diagnosis of Alzheimer's-type and multi-infarct dementia. A pilot study," Archives of General Psychiatry, vol. 44, no. 11, pp. 993998, 1987.

[83] A. F. Leuchter, T. F. Newton, I. A. Cook, D. O. Walter, S. Rosenberg-Thompson, and P. A. Lachenbruch, "Changes in brain functional connectivity in Alzheimer-type and multiinfarct dementia," Brain, vol. 115, no. 5, pp. 1543-1561, 1992.

[84] V. Jelic, S. E. Johansson, O. Almkvist et al., "Quantitative electroencephalography in mild cognitive impairment: longitudinal changes and possible prediction of Alzheimer's disease," Neurobiology of Aging, vol. 21, no. 4, pp. 533-540, 2000.

[85] O. Almkvist, V. Jelic, K. Amberla, E. Hellström-Lindahl, L. Meurling, and A. Nordberg, "Responder characteristics to a single oral dose of cholinesterase inhibitor: a double-blind placebo-controlled study with tacrine in Alzheimer patients," Dementia and Geriatric Cognitive Disorders, vol. 12, no. 1, pp. 22-32, 2001.

[86] M. Brunovsky, M. Matousek, A. Edman, K. Cervena, and V. Krajca, "Objective assessment of the degree of dementia by 
means of EEG," Neuropsychobiology, vol. 48, no. 1, pp. 19-26, 2003.

[87] C. Babiloni, G. Frisoni, F. Vecchio et al., "Global functional coupling of resting EEG rhythms is abnormal in mild cognitive impairment and Alzheimer's disease: a multicenter EEG study," Journal of Psychophysiology, vol. 23, no. 4, pp. 224-234, 2009.

[88] C. J. Stam, "Nonlinear dynamical analysis of EEG and MEG: review of an emerging field," Clinical Neurophysiology, vol. 116, no. 10, pp. 2266-2301, 2005.

[89] C. Besthorn, H. Sattel, C. Geiger-Kabisch, R. Zerfass, and H. Forstl, "Parameters of EEG dimensional complexity in Alzheimer's disease," Electroencephalography and Clinical Neurophysiology, vol. 95, no. 2, pp. 84-89, 1995.

[90] J. Jeong, S. Y. Kim, and S. H. Han, "Non-linear dynamical analysis of the EEG in Alzheimer's disease with optimal embedding dimension," Electroencephalography and Clinical Neurophysiology, vol. 106, no. 3, pp. 220-228, 1998.

[91] C. Babiloni, R. Ferri, D. V. Moretti et al., "Abnormal frontoparietal coupling of brain rhythms in mild Alzheimer's disease: a multicentric EEG study," European Journal of Neuroscience, vol. 19, pp. 2583-2590, 2004.

[92] Y. A. L. Pijnenburg, V. D. Y. Made, A. M. van Cappellen van Walsum, D. L. Knol, PH. Scheltens, and C. J. Stam, "EEG synchronization likelihood in mild cognitive impairment and Alzheimer's disease during a working memory task," Clinical Neurophysiology, vol. 115, no. 6, pp. 1332-1339, 2004.

[93] C. J. Stam, A. M. van Cappellen van Walsum, Y. A. L. Pijnenburg et al., "Generalized synchronization of MEG recordings in Alzheimer's disease: evidence for involvement of the gamma band," Journal of Clinical Neurophysiology, vol. 19, no. 6, pp. 562-574, 2002.

[94] M. Kikuchi, Y. Wada, Y. Koshino, Y. Nanbu, and T. Hashimoto, "Effects of scopolamine on interhemispheric EEG coherence in healthy subjects: analysis during rest and photic stimulation," Clinical EEG Electroencephalography, vol. 31, no. 2, pp. 109-115, 2000.

[95] A. E. P. Villa, I. V. Tetko, P. Dutoit, and G. Vantini, "Nonlinear cortico-cortical interactions modulated by cholinergic afferences from the rat basal forebrain," BioSystems, vol. 58, no. 1-3, pp. 219-228, 2000.

[96] L. A. Coben, W. Danziger, and M. Storandt, "A longitudinal EEG study of mild senile dementia of Alzheimer type: changes at 1 year and at 2.5 years," Electroencephalography and Clinical Neurophysiology, vol. 61, no. 2, pp. 101-112, 1985.

[97] H. Soininen, J. Partanen, V. Laulumaa, E. L. Helkala, M. Laakso, and P. J. Riekkinen, "Longitudinal EEG spectral analysis in early stage of Alzheimer's disease," Electroencephalography and Clinical Neurophysiology, vol. 72, no. 4, pp. 290-297, 1989.

[98] M. Mesulam, “The cholinergic lesion of Alzheimer's disease: pivotal factor or side show?” Learning and Memory, vol. 11, no. 1, pp. 43-49, 2004.

[99] D. P. Holschneider, A. F. Leuchter, O. U. Scremin, D. M. Treiman, and N. Y. Walton, "Effects of cholinergic deafferentation and NGF on brain electrical coherence," Brain Research Bulletin, vol. 45, no. 5, pp. 531-541, 1998.

[100] Y. Tanaka, H. Hanyu, H. Sakurai, M. Takasaki, and K. Abe, "Atrophy of the substantia innominata on magnetic resonance imaging predicts response to donepezil treatment in Alzheimer's disease patients," Dementia and Geriatric Cognitive Disorders, vol. 16, no. 3, pp. 119-125, 2003.
[101] G. Rodriguez, P. Vitali, C. De Leo, F. De Carli, N. Girtler, and F. Nobili, "Quantitative EEG changes in Alzheimer patients during long-term donepezil therapy," Neuropsychobiology, vol. 46, no. 1, pp. 49-56, 2002.

[102] J. A. H. R. Claassen and R. W. M. M. Jansen, "Cholinergically mediated augmentation of cerebral perfusion in Alzheimer's disease and related cognitive disorders: the cholinergicvascular hypothesis," Journals of Gerontology, vol. 61, no. 3, pp. 267-271, 2006.

[103] B. Winblad, K. Palmer, M. Kivipelto et al., "Mild cognitive impairment-beyond controversies, towards a consensus: report of the International Working Group on Mild Cognitive Impairment," Journal of Internal Medicine, vol. 256, no. 3, pp. 240-246, 2004.

[104] S. Gauthier, B. Reisberg, M. Zaudig et al., "International psychogeriatric association expert conference on mild cognitive impairment. Mild cognitive impairment," Lancet, vol. 367, pp. 1262-1270, 2006.

[105] F. Portet, P. J. Ousset, P. J. Visser et al., "Mild cognitive impairment (MCI) in medical practice: a critical review of the concept and new diagnostic procedure. Report of the MCI Working Group of the European Consortium on Alzheimer's Disease," Journal of Neurology, Neurosurgery and Psychiatry, vol. 77, no. 6, pp. 714-718, 2006.

[106] P. M. Rossini, C. Del Percio, P. Pasqualetti et al., "Conversion from mild cognitive impairment to Alzheimer's disease is predicted by sources and coherence of brain electroencephalography rhythms," Neuroscience, vol. 143, no. 3, pp. 793-803, 2006.

[107] V. Jelic, M. Shigeta, P. Jnlin, O. Almkvist, B. Winblad, and L. O. Wahhmd, "Quantitative electroencephalography power and coherence in Alzheimer's disease and mild cognitive impairment," Dementia, vol. 7, no. 6, pp. 314-323, 1996.

[108] M. Grunwald, F. Busse, A. Hensel et al., "Correlation between cortical $\theta$ activity and hippocampal volumes in health, mild cognitive impairment, and mild dementia," Journal of Clinical Neurophysiology, vol. 18, no. 2, pp. 178-184, 2001.

[109] Y. T. Kwak, "Quantitative EEG findings in different stages of Alzheimer's disease," Journal of Clinical Neurophysiology, vol. 23, no. 5, pp. 456-461, 2006.

[110] E. Arnáiz and O. Almkvist, "Neuropsychological features of mild cognitive impairment and preclinical Alzheimer's disease," Acta Neurologica Scandinavica, Supplement, vol. 107, no. 179, pp. 34-41, 2003.

[111] G. Rodriguez, P. Vitali, M. Canfora et al., "Quantitative EEG and perfusional single photon emission computed tomography correlation during long-term donepezil therapy in Alzheimer's disease," Clinical Neurophysiology, vol. 115, no. 1, pp. 39-49, 2004.

[112] C. Babiloni, L. Benussi, G. Binetti et al., "Apolipoprotein E and alpha brain rhythms in mild cognitive impairment: a multicentric electroencephalogram study," Annals of Neurology, vol. 59, no. 2, pp. 323-334, 2006.

[113] C. Babiloni, L. Benussi, G. Binetti et al., "Genotype (cystatin C) and EEG phenotype in Alzheimer disease and mild cognitive impairment: a multicentric study," NeuroImage, vol. 29, no. 3, pp. 948-964, 2006.

[114] H. Wolf, V. Jelic, H. J. Gertz, A. Nordberg, P. Julin, and L. O. Wahlund, "A critical discussion of the role of neuroimaging in mild cognitive impairment," Acta Neurologica Scandinavica, Supplement, vol. 107, no. 179, pp. 52-76, 2003.

[115] A. Fernández, J. Arrazola, F. Maestú et al., "Correlations of hippocampal atrophy and focal low-frequency magnetic 
activity in Alzheimer disease: volumetric MR imagingmagnetoencephalographic study," American Journal of Neuroradiology, vol. 24, no. 3, pp. 481-487, 2003.

[116] E. L. Helkala, T. Hänninen, M. Könönen et al., "Slow-wave activity in the spectral analysis of the electroencephalogram and volumes of hippocampus in subgroups of Alzheimer's disease patients," Behavioral Neuroscience, vol. 110, no. 6, pp. 1235-1243, 1996.

[117] A. Spiegel, P. H. Tonner, and M. Renna, "Altered states of consciousness: processed EEG in mental disease," Best Practice and Research: Clinical Anaesthesiology, vol. 20, no. 1, pp. 57-67, 2006.

[118] L. S. Prichep, E. R. John, S. H. Ferris et al., "Prediction of longitudinal cognitive decline in normal elderly with subjective complaints using electrophysiological imaging," Neurobiology of Aging, vol. 27, no. 3, pp. 471-481, 2006.

[119] M. Nuwer, "Assessment of digital EEG, quantitative EEG, and EEG brain mapping: report of the American Academy of Neurology and the American Clinical Neurophysiology Society," Neurology, vol. 49, no. 1, pp. 277-292, 1997.

[120] J. J. Claus, R. L. M. Strijers, E. J. Jonkman et al., "The diagnostic value of electroencephalography in mild senile Alzheimer's disease," Clinical Neurophysiology, vol. 110, no. 5, pp. 825-832, 1999.

[121] K. Bennys, G. Rondouin, C. Vergnes, and J. Touchon, "Diagnostic value of quantitative EEG in Alzheimer's disease," Neurophysiologie Clinique, vol. 31, no. 3, pp. 153-160, 2001.

[122] S. Brassen, D. F. Braus, W. Weber-Fahr, H. Tost, S. Moritz, and G. Adler, "Late-onset depression with mild cognitive deficits: electrophysiological evidences for a preclinical dementia syndrome," Dementia and Geriatric Cognitive Disorders, vol. 18, no. 3-4, pp. 271-277, 2004.

[123] C. Lehmann, T. Koenig, V. Jelic et al., "Application and comparison of classification algorithms for recognition of Alzheimer's disease in electrical brain activity (EEG)," Journal of Neuroscience Methods, vol. 161, no. 2, pp. 342-350, 2007.

[124] P. Missonnier, G. Gold, F. R. Herrmann et al., "Decreased theta event-related synchronization during working memory activation is associated with progressive mild cognitive impairment," Dementia and Geriatric Cognitive Disorders, vol. 22, no. 3, pp. 250-259, 2006.

[125] M. Buscema, P. Rossini, C. Babiloni, and E. Grossi, "The IFAST model, a novel parallel nonlinear EEG analysis technique, distinguishes mild cognitive impairment and Alzheimer's disease patients with high degree of accuracy," Artificial Intelligence in Medicine, vol. 40, no. 2, pp. 127-141, 2007. 


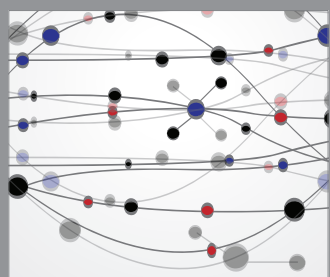

The Scientific World Journal
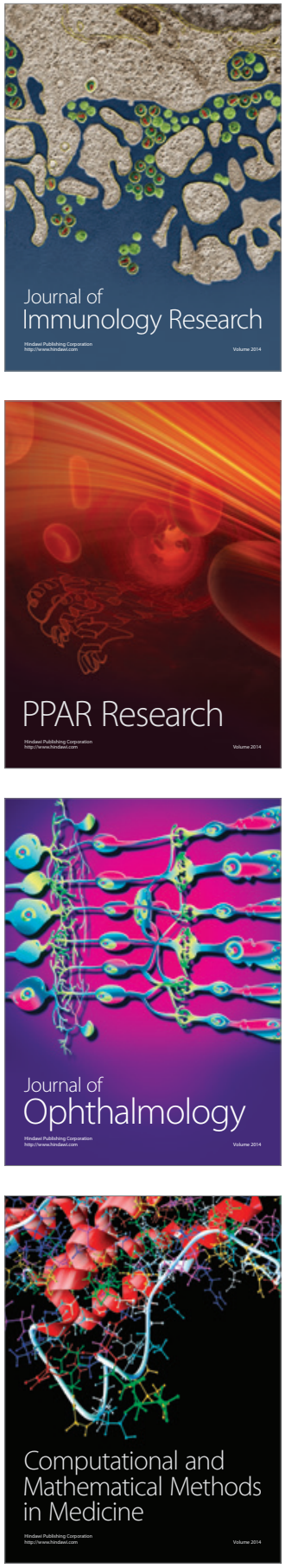

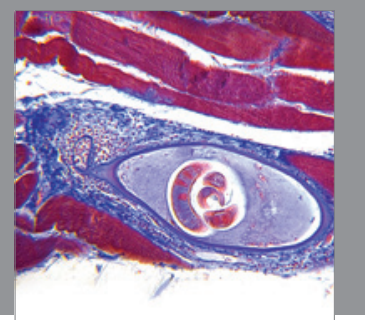

Gastroenterology

Research and Practice
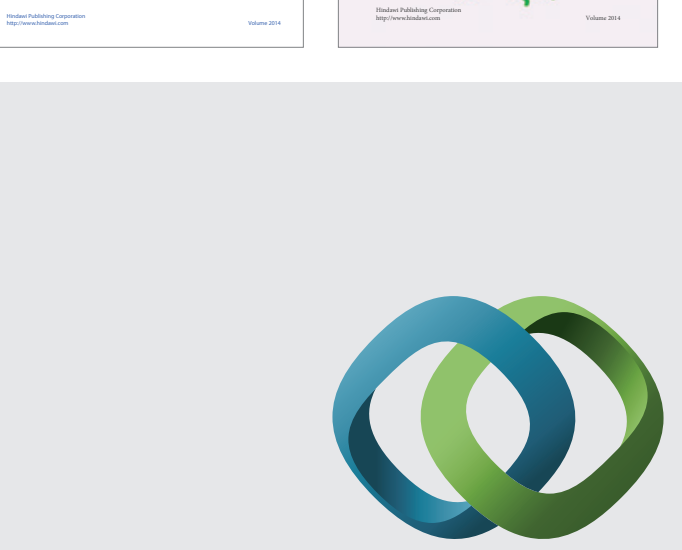

\section{Hindawi}

Submit your manuscripts at

http://www.hindawi.com
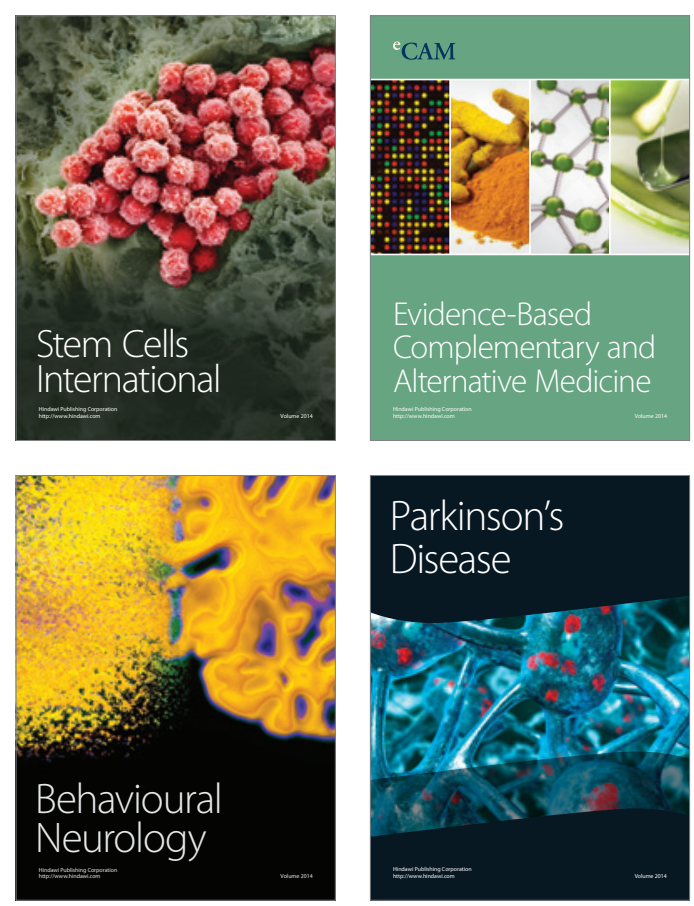

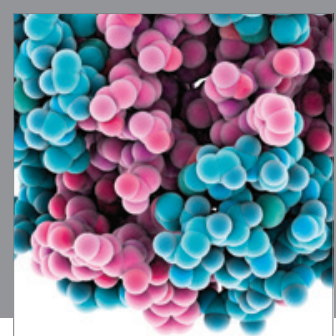

Journal of
Diabetes Research

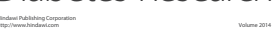

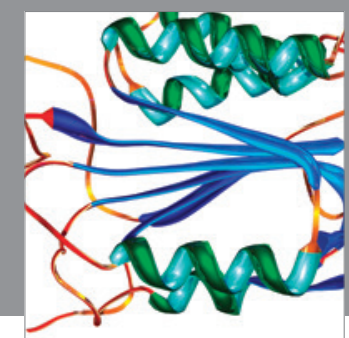

Disease Markers
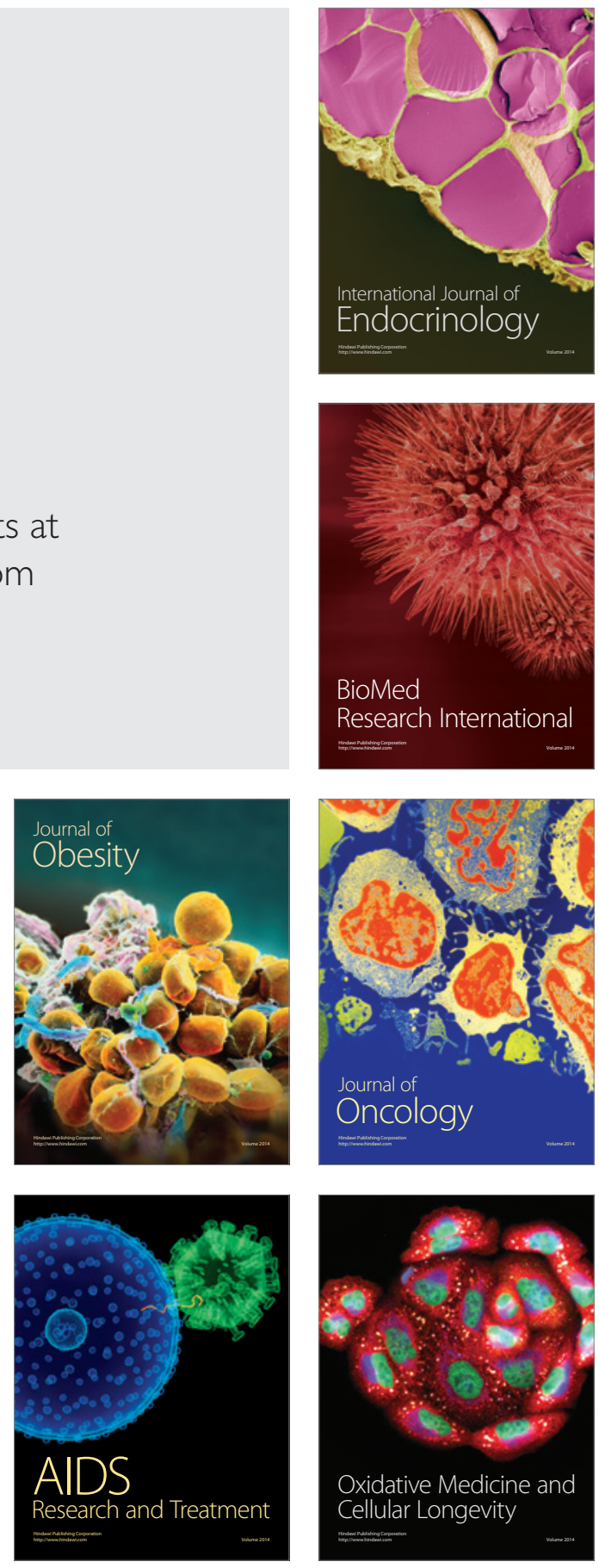